\title{
A Short Hairpin Loop-Structured Oligodeoxynucleotide Targeting the Virion-Associated RNase H of HIV Inhibits HIV Production in Cell Culture and in huPBL-SCID Mice
}

\author{
Jochen Heinrich $^{a} \quad$ Dominique Schols $^{b} \quad$ Karin Moelling ${ }^{a, c}$ \\ ${ }^{a}$ University of Zurich, Zurich, Switzerland; ${ }^{b}$ Rega Institute for Medical Research, K.U. Leuven, Leuven, Belgium; \\ ${ }^{\mathrm{c}}$ Max Planck Institute of Molecular Genetics, Berlin, Germany
}

\section{Key Words}

Oligodeoxynucleotide $\cdot$ HIV $\cdot$ huPBL-SCID mice

\begin{abstract}
Background: We have recently demonstrated that an oligodeoxynucleotide (ODN) can enter HIV particles and form a local hybrid at the highly conserved polypurine tract (PPT), the target site of the ODN. This hybrid is recognized by the retrovirus-specific RNase $\mathrm{H}$, which is a virion-associated enzyme. It cleaves the RNA at local hybrids and thereby destroys viral infectivity. This mechanism has been described previously in a mouse model using an oncogenic retrovirus and was commented as driving HIV into suicide. The RNase $\mathrm{H}$ is one of four retrovirus-specific enzymes and not yet targeted by antiviral drugs. Aims: We wanted to analyze the tendency of ODNs to induce mutations in cell culture and its efficacy to inhibit HIV in humanized SCID mice. Method: We used cultures of CD4+ T cells infected with HIV-1 after serial passage in the presence of ODNs in the supernatant for up to 3 months, using Foscarnet as positive control, and treated HIV-infected huPBL-SCID mice repeatedly with ODN. $\boldsymbol{R e}$ -
\end{abstract}

sults: Treatment with ODN did not induce mutations of the PPT or the reverse transcriptase polymerase domain in vitro, whereas Foscarnet did. We furthermore demonstrate that ODNs inhibit HIV-1 replication in humanized HIV-infected SCID mice.

Copyright $\odot 2011$ S. Karger AG, Basel

HIV encodes four enzymes, three of which are the major targets of antiviral therapy [1]. Recently, we described another approach which exploits the virion-associated $\mathrm{RNase} H$ activity, which is fused to the viral reverse transcriptase (RT). The RNase $\mathrm{H}$ is a virion-associated retroviral enzyme which has been shown to specifically cleave RNA in RNA-DNA hybrids [2]. An oligodeoxynucleotide (ODN) can enter virus particles, form a local hybrid and the bound RNA is cleaved by the RNase H, which destroys viral infectivity [3]. The ODN is a double-

J.H. and D.S. contributed equally to this work.

\section{KARGER}

Fax +4161306 1234 E-Mail karger@karger.ch www.karger.com
(C) 2011 S. Karger AG, Basel

0300-5526/12/0553-0242\$38.00/0

Accessible online at:

www.karger.com/int
Karin Moelling

Max Planck Institute of Molecular Genetics

Ihnestrasse 73

DE-14195 Berlin (Germany)

Tel. +49 308311 595, E-Mail moelling@imm.uzh.ch 


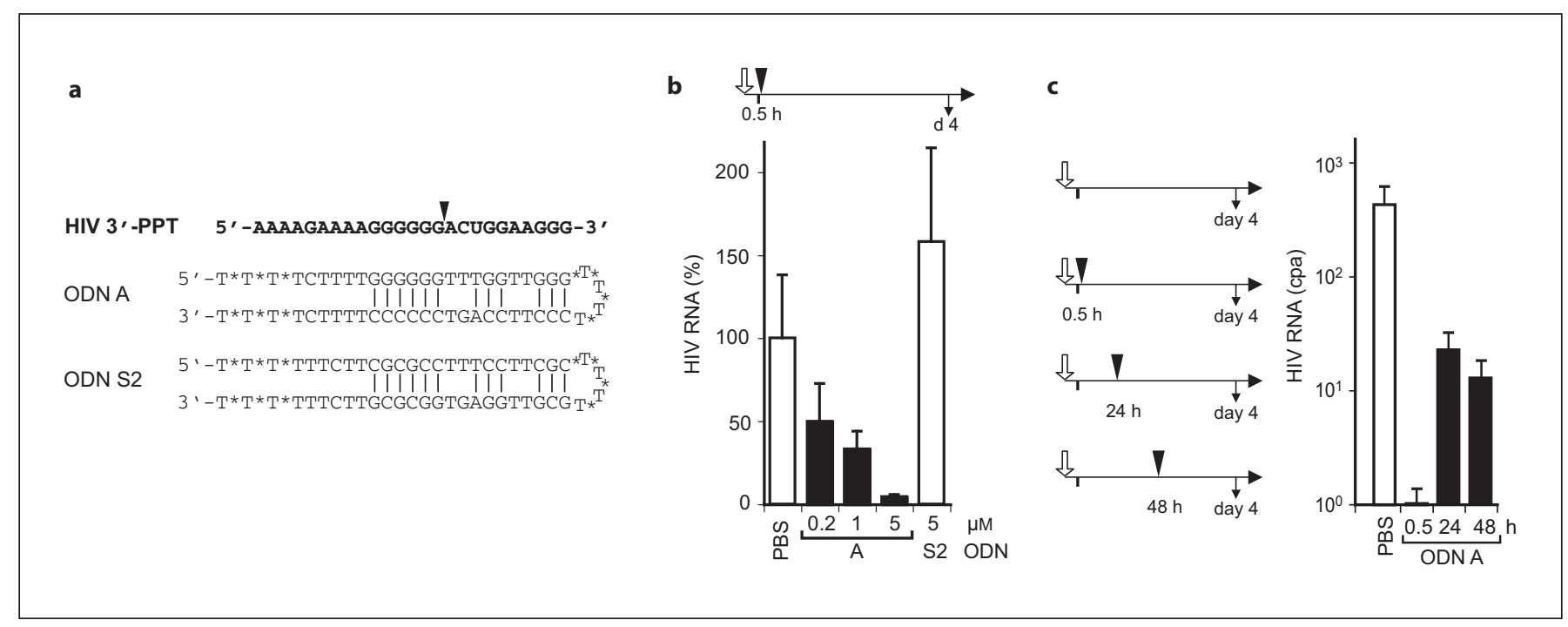

Fig. 1. ODN A inhibits HIV in vitro. a Sequences of the HIV-1 $3^{\prime}$-PPT and ODNs used. The cleavage site of RNase $\mathrm{H}$ is indicated by an arrowhead and phosphothioates by asterisks. b Dose-dependent inhibition of HIV-1 by ODN A. C81-66/45 cells $\left(1 \times 10^{5} /\right.$ $\mathrm{ml}$ ) were infected with HIV-1 (open arrow, multiplicity of infection $=0.02$ ) for $30 \mathrm{~min}$ at $37^{\circ} \mathrm{C}$, then washed (vertical line) and seeded in 48-well dishes prefilled with medium and ODN A (closed arrowhead) to achieve the indicated final concentration. At day 4 supernatants were harvested and viral titers determined

stranded, hairpin loop-structured 54mer ODN A (fig. 1a), one strand of which is fully complementary to the $3^{\prime}$ polypurine tract (PPT) of HIV RNA. ODN A contains a second strand partially complementary to the antisense strand, which protects against nucleases and enables cellular entry (fig. 1a). Both strands are connected by a linker of four thymidines. The linker and the three terminal nucleotides at each end are modified by phosphothioates. ODN A inhibits HIV in cell culture [3-8] and in plasma of HIV-infected patients during ex vivo treatment [9]. In a retrovirus mouse model with spleen focus-forming virus (SFFV) we used an ODN targeting the PPT of SFFV, and demonstrated reduction of the virus load in plasma, reduction of disease progression and prevention of infection [6]. Furthermore, using a recombinant lentiviral vector containing the HIV-PPT, we recently showed that ODN A was able to reduce lentivirus titers in the mouse vagina therapeutically and prophylactically [10]. ODNs can also inhibit replication of viruses such as influenza A in a mouse model [11], herpes simplex viruses 1 and 2 [12], hepatitis viruses $\mathrm{C}$ and $\mathrm{B}$ (in preparation) or expression of oncogenes by using cellular RNases $\mathrm{H}$ or other mechanisms such as translational arrest $[13,15]$.

ODN Inhibiting HIV Production in Cell

Culture and huPBL-SCID Mice by gag-specific qRT-PCR (vertical arrow). Bars represent the means of relative HIV RNA levels in percent + SE of an experiment performed in triplicates. Bars represent the mean of HIV RNA levels in copies per assay (cpa). c Treatment at different time points was performed as indicated in the scheme. At later time points ODN A was directly added to the infected cells. Bars represent HIV RNA levels in cpa. The standard deviation is typically around $20 \%$.

The dose dependence of the antiviral activity of ODN A on HIV in the human $\mathrm{T}$ cell line C81/66-45 is shown in figure $1 \mathrm{~b}$. The cells were infected with HIV-1 IIIB, which was used throughout the study, for $30 \mathrm{~min}$, washed, treated with three concentrations of $\operatorname{ODN~A}(0.2,1$ and $5 \mu \mathrm{M}$ final concentration) or the scrambled control ODN S2 (5 $\mu \mathrm{M})$, and viral growth was allowed to continue for 4 days at $37^{\circ} \mathrm{C}$ (fig. 1b). Viral RNA was determined in the supernatant by gag-specific quantitative RT-PCR [9]. ODN A inhibited viral growth with a half maximal inhibitory concentration of approximately $200 \mathrm{nM}$, while $5 \mu \mathrm{M}$ of the control did not. We then added the ODNs at later time points, 24 and $48 \mathrm{~h}$ instead of $0.5 \mathrm{~h}$ (fig. 1c). The viral RNA levels were reduced approximately 500 -fold at $0.5 \mathrm{~h}$, whereas later only 20 - to 30 -fold. The later time points allow virus replication, and the ratio of ODN to virus is then much lower. The effect of ODN is dose dependent.

To further characterize the ODN we analyzed the effect of ODN A on HIV-1 virus growth in two systems, first by cell culture with serially passaging of $20 \%$ of the supernatant to new cell cultures in the presence of ODNs and then by treatment of the virus in humanized mice.

Intervirology 2012;55:242-246 
Fig. 2. Serial passages of HIV-1 with treatment with ODN A. a Short-term serial passage. Infections, ODN A treatment and analysis were performed in triplicates as described in figure $1 \mathrm{~b}$ without washing after infection. Twenty percent of the harvested supernatant was used for the next round of infection. The graph represents the viral titer in supernatants of the indicated passage for continuous (left panel) or discontinuous treatment (right panel) as indicated. The individual series are indicated by several Ts and Os corresponding to the passages with and without treatments, respectively. $\mathbf{b}$ Serial passages and analysis of mutants. Serial passages were performed for 3 months comprising 16 passages with few modifications as described in a, except that $50 \%$ of the supernatant was used for the next passage, and a second spike of ODN A was applied $4 \mathrm{~h}$ after infection (not shown). ODN A was applied at $5 \mu \mathrm{M}$ and Foscarnet at $100 \mu \mathrm{M}$. Viruses from passages $1,5,7,10$ and 14 were analyzed by population sequencing for mutations in the target regions. The scheme indicates the sequenced 3 -PPT $(8,615-8,639)$, region $(8,511-8,672)$ and the polymerase domain segment pol $(1,697-2,875)$. Coordinates refer to the genomic HIV-1 NCBI reference sequence NC_001802.1. Known mutations characteristic for resistance against Foscarnet are indicated by single letter amino acids followed by position and the amino acid replacement. Of these mutations we boxed the ones found in this study. c Summary of mutations. The table summarizes treatment, target, sequenced regions and mutations observed here for the indicated passages. $-=$ No mutation detected; n.d. $=$ not done.

We allowed ODNs to be present at 0.1 or $2.5 \mu \mathrm{M}$ concentration and used PBS as control (fig. $2 \mathrm{a}$, left panel). We observed a rapid strong decrease in the viral load at 2.5 $\mu \mathrm{M}$ ODN A by several logs, also the lower concentration caused a less prominent reduction of viral load and 2-4 passages were sufficient to drastically reduce the viral load. The administration of ODN A could even be transiently omitted for 1 or 2 passages (fig. 2a, right panel). After passage 2 with 2 treatments (TT), virus load was down but increased in the absence of treatment (TT000). This increase was not due to resistance, because resumed treatment (TT00T) could inhibit it. To test resistance after a higher number of passages, we increased the volume of transferred supernatants to $50 \%$, because otherwise

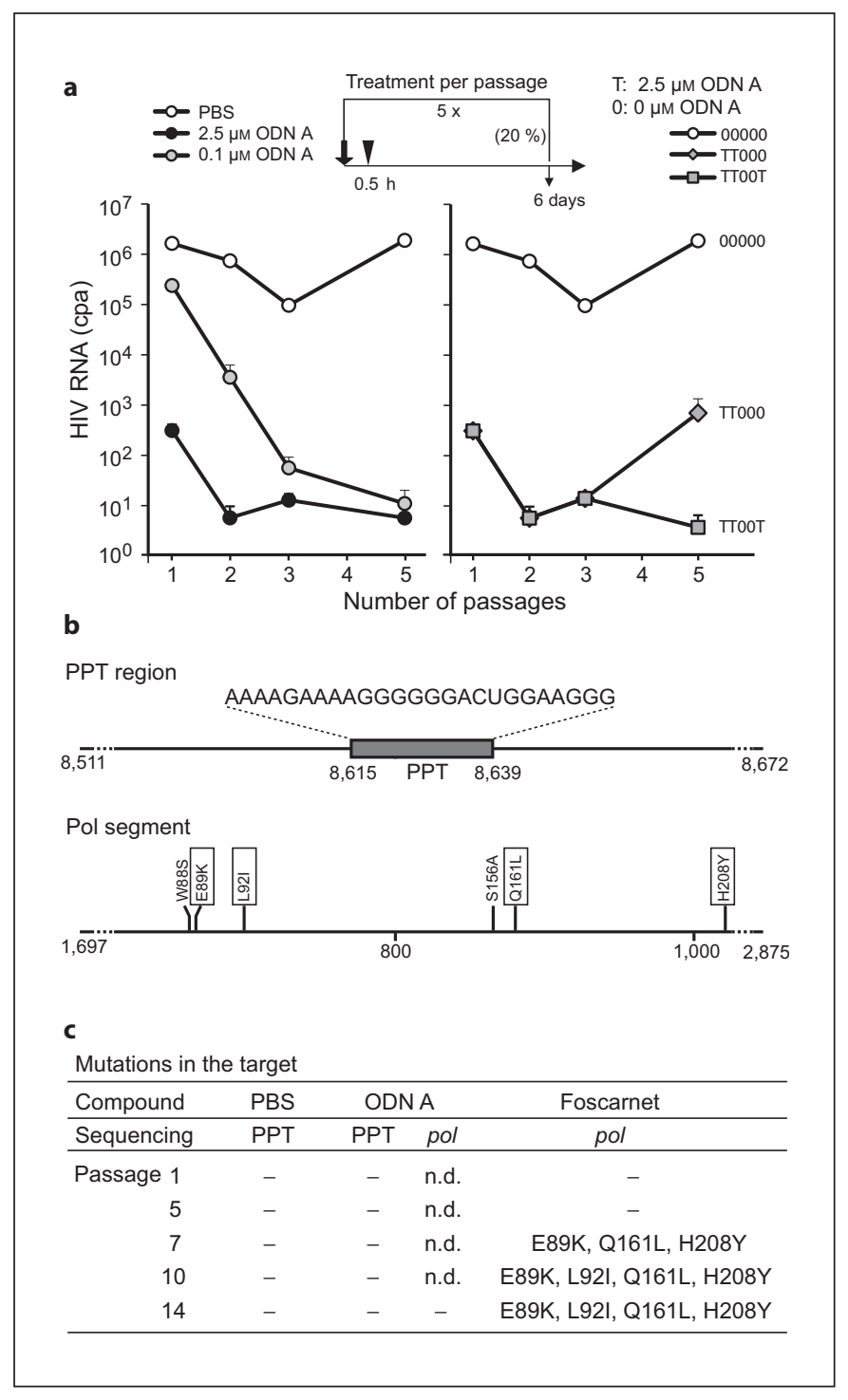

there was not enough virus left. In the presence of ODN A we could maintain the cultures for up to 14 passages, corresponding to 3 months. For comparison, the highly mutagenic RT inhibitor Foscarnet was used as positive and PBS as negative control. Samples of passages 1, 5, 7, 10 and 14 were analyzed for nucleotide variations in the 3 ' PPT region and the region coding for the polymerase domain (pol) of RT (fig. 2b). We did not detect any mutants in both regions for ODN A and PBS treatment (fig. 2c). In contrast, upon treatment with Foscarnet, specific mutations in the pol region appeared already at passage 7. Thus, ODN A did not as quickly induce mutations, which can be attributed to the fact that cleavage of the viral RNA is an all or none effect and not easily mutated. 
Fig. 3. Inhibition of HIV-1 in vivo. a SCID mice were injected with huPBL at day 0 (open arrow) and colonization of the mice was allowed for two weeks (as described previously). At day $15 \mathrm{HIV}-1$ IIIB (thick arrow, $1 \times 10^{4} 50 \%$ tissue culture-infective dose) and ODN A (filled triangle) were mixed and injected i.p. together (line on top). ODN A was injected i.p. every one or two days as indicated (filled triangles). At day 28 blood samples were taken, plasma was prepared and p24 Ag levels were measured by specific p24 Ag ELISA (Perkin-Elmer). b The three groups tested were HIVinfected mice treated with ODN A $(n=6)$ or PBS $(n=3)$ as control, and uninfected untreated mice $(\mathrm{n}=4)$. The p24 levels are shown $(-=$ not detected and below $<5 \mathrm{pg} / \mathrm{ml}$ detection limit). $\mathbf{c}$ The graph indicates the mean p24 levels \pm SE for PBS- and ODN A-treated HIV-1-infected mice. ${ }^{*} \mathrm{p}<0.05$.

Thus, we demonstrated here that ODN A inhibits growth of HIV in vitro in a single passage up to 100 - or 500 -fold. More than $10^{5}$-fold reduction can be achieved in a few passages, and the virus remained equally sensitive even after prolonged treatments (fig. 2), exhibiting 10 -fold reduction of virus titers in untreated and ODN A-treated cultures (data not shown). Foscarnet gave rise to mutations in the polymerase domain under these conditions. We attribute these strong inhibitory effects to destruction of viral infectivity in the supernatant during medium transfer by each passage. Destruction of viral RNA is different from binding of a drug.

The ODN effect in cell culture is indicative for what may happen in animals. This is of high interest but difficult to achieve, since there are no simple small animal models. We had tested SFFV in mice and lentiviral vector particles in the mouse vagina $[6,10]$. For this study we were able to test viral growth of HIV-1 in the huPBLSCID mouse model [16]. Based on the results described above and published previously [6] we designed a regimen which ensured a high ODN A concentration during the test period by continuous treatment. HIV-1 and ODN A were mixed and the mixture was immediately intraperitoneally (i.p.) applied at day 15 after engrafting SCID mice with huPBL, followed by i.p. administration of ODN A every 1-2 days up to day 28, when HIV-1 is detectable in the plasma of HIV-1-infected huPBL-SCID mice (fig. 3a). Three groups were used: HIV-infected huPBLSCID mice treated with ODN A $(n=6)$ or PBS $(n=3)$ as

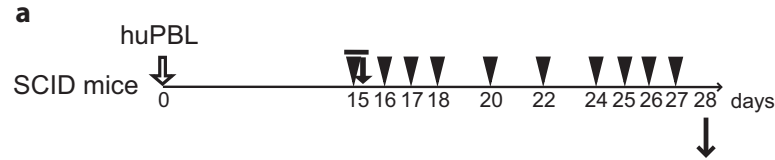

b

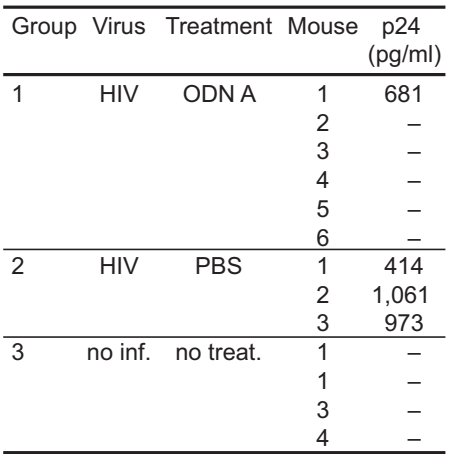

c

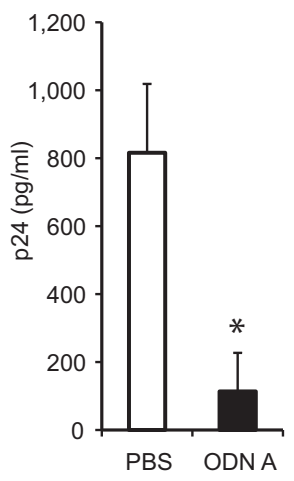

negative control as well as uninfected, untreated huPBLSCID mice $(n=4)$. No visible toxicity or unusual behavior or appearance was observed for any of the mice. At day 28 the mice were sacrificed and the p24 HIV-1 Ag levels in plasma were determined by a specific p24 ELISA (fig. 3b, c). All 3 HIV-1-infected mice treated with PBS exhibited detectable levels of p24 HIV-1 Ag corresponding to the expected infection rate of $100 \%$. Mice infected with HIV-1 and treated with ODN A did not allow any virus detection in 5 of 6 cases. Only in 1 of 6 mice was the virus detectable, corresponding to an apparent infection rate of $17 \%$, demonstrating that ODN A dramatically reduced HIV-1 titers in this in vivo humanized mouse model.

We conclude that ODN A inhibited HIV replication in vivo in an huPBL-SCID mouse model without any visible sign of toxicity, confirming our previous results about oligonucleotide-mediated reduction of SFFV and self-inactivating lentiviral vectors in vivo. We applied the ODN repeatedly, but it may be a possible explanation that the virus was predominantly killed at the very beginning. Once the virus escaped this early treatment and replicated, it could no longer be controlled in 1 of the 6 treated mice. We have noticed similar responses with SFFV in mice and prevention of infection by a single very early treatment [6]. Since we are specifically inhibiting the cell-free virus particles with ODNs, application could be pre-exposure prophylaxis (PrEP), for example, in the form of a microbicide against sexual transmission of 
HIV in the Third World. ODNs targeted to virions are the only compounds known to specifically destroy virus before infection. This may also be of benefit for prevention of mother-to-child transmission and therapeutic treatment.

\section{Acknowledgements}

We thank Dr. Srikant Mathur for initial serial passages and population sequencing and gratefully acknowledge Dr. Cyril Shah for help with sequencing. We are grateful to Sandra Claes and Eric Fonteyn for excellent technical assistance. This work was supported by Centers of Excellence of the K.U. Leuven (EF/05/015).

\section{References}

1 De Clercq E: The design of drugs for HIV and HCV. Nat Rev Drug Discov 2007;6:10011018 .

$\checkmark 2$ Moelling K: Characterization of reverse transcriptase and $\mathrm{RNase} \mathrm{H}$ from friend-murine leukemia virus. Virology 1974;62:4659.

3 Matskevich AA, Ziogas A, Heinrich J, Quast S, Moelling K: Short partially double-stranded oligodeoxynucleotide induces reverse transcriptasemediated cleavage of HIV RNA and abrogates infectivity of virions. AIDS Res Hum Retroviruses 2006;22:1220-1230.

$\checkmark 4$ Jendis J, Strack B, Moelling K: Inhibition of replication of drug-resistant HIV type 1 isolates by polypurine tract-specific oligodeoxynucleotide TFO A. AIDS Res Hum Retroviruses 1998;14:999-1005.

5 Jendis J, Strack B, Volkmann S, Boni J, Moelling K: Inhibition of replication of fresh HIV type 1 patient isolates by a polypurine tractspecific self-complementary oligodeoxynucleotide. AIDS Res Hum Retroviruses 1996; 12:1161-1168.
-6 Matzen K, Elzaouk L, Matskevich A Nitzsche A, Heinrich J, Moelling K: RNase $\mathrm{H}$-induced suicide of a retrovirus by oligodeoxynucleotides in a mouse model. Nat Biotechnol 2007;25:669-674.

7 Moelling K, Abels S, Jendis J, Matskevich A, Heinrich J: Silencing of HIV by hairpinloop-structured DNA oligonucleotide. FEBS Lett 2006;580:3545-3550.

$>8$ Volkmann S, Jendis J, Frauendorf A, Moelling $\mathrm{K}$ : Inhibition of $\mathrm{HIV}-1$ reverse transcription by triple-helix forming oligonucleotides with viral RNA. Nucleic Acids Res 1995;23:1204-1212.

-9 Heinrich J, Mathur S, Matskevich AA, Moelling K: Oligonucleotide-mediated retroviral RNase $\mathrm{H}$ activation leads to reduced HIV-1 titer in patient-derived plasma. AIDS 2009; 23:213-221.

10 Wittmer-Elzaouk L, Jung-Shiu J, Heinrich J, Moelling K: Retroviral selfinactivation in the mouse vagina induced by short DNA. Antiviral Res 2009;82:22-28.

$\checkmark 11$ Kwok T, Helfer H, Alam MI, Heinrich J, Pavlovic J, Moelling K: Inhibition of Influenza A virus replication by short double-stranded oligodeoxynucleotides. Arch Virol 2009; 154:109-114.
12 Falkenhagen A, Heinrich J, Moelling K: Short hairpin-loop-structured oligodeoxynucleotides reduce HSV-1 replication. Virol J 2009;6:43.

-13 Hofmann MH, Heinrich J, Radziwill G, Moelling K: A short hairpin DNA analogous to miR-125b inhibits C-Raf expression, proliferation, and survival of breast cancer cells. Mol Cancer Res 2009;7:1635-1644.

14 Kwok T, Heinrich J, Jung-Shiu J, Meier MG, Mathur S, Moelling K: Reduction of gene expression by a hairpin-loop structured oligodeoxynucleotide: alternative to siRNA and antisense. Biochim Biophys Acta 2009;1790: 1170-1178.

15 Noreen F, Heinrich J, Moelling K: Antitumor activity of small double-stranded oligodeoxynucleotides targeting telomerase RNA in malignant melanoma cells. Oligonucleotides 2009;19:169-178.

16 Balzarini J, Pelemans H, Aquaro S, Perno CF, Witvrouw M, Schols D, De Clercq E, Karlsson A: Highly favorable antiviral activity and resistance profile of the novel thiocarboxanilide pentenyloxy ether derivatives UC-781 and UC-82 as inhibitors of human immunodeficiency virus type 1 replication. Mol Pharmacol 1996;50:394-401. 